\title{
Correlative Isotopic Analysis by Image Fusion of Electron Microscopy and Secondary Ion Mass Spectrometry Data
}

\author{
Jay G. Tarolli ${ }^{1}$, Benjamin E. Naes ${ }^{1}$, and David Willingham ${ }^{1}$ \\ 1. Pacific Northwest National Laboratory, Richland, WA, USA
}

Image fusion is a technique to combine complementary information from two or more input images into a final image which, by definition, better describes the scene than any of the original images could alone. Capable of presenting valuable information from multiple sources in a single visualization, image fusion has been successfully applied to a variety of disciplines, including satellite [1] and medical imaging [2]. Recently, the technique has been developed for application to new analytical techniques, most significantly to combine chemical information from secondary ion mass spectrometry (SIMS) images with images acquired using electron microscopy [3, 4].

The basis for image fusion with SIMS centers on the types of information acquired using different imaging modalities and the achievable lateral resolution of the respective techniques. SIMS is an excellent tool for mapping the isotopic and elemental distributions of complex systems. However, the lateral resolution is limited by the primary ion probe and other instrumental characteristics, and is often substandard when compared to that of electron microscopes. These techniques, while providing superior resolution and, thus, images which can resolve smaller features and domains than their SIMS counterpart, lack welldefined elemental and no isotopic information. Therefore, the fusion of spatially resolved information with isotopic and elemental information allows for new systems with smaller features to be analyzed with the benefits afforded by SIMS.

In order to accomplish image fusion of SIMS and electron microscope images, the two images must first be properly registered, meaning that the same exact analysis area is presented in both images. Expanding image fusion to two imaging modalities which are located in different facilities, such as is often the case with SIMS and electron microscopy, requires a well-developed, systematic protocol for acquiring the input images. This may include reference points on the sample to ensure roughly the same area is analyzed in both instruments. Registration is then accomplished using an iterative process to determine a transform which maps one input image into the image space of the other with the highest degree of similarity. Quantifying the similarity between two images could be based on a comparison of pixel intensities across the images or on the location of user-defined control points which are representative of physical features present in both images. When distinct features or markers are present in both images, the latter option is preferred since the registration process is more efficient.

The goal is to create a standardized methodology for acquiring, pre-processing, and fusing an elemental/isotopic image with a higher resolution electron microscopy image. Initially, a pre-fabricated image standard consisting of a tantalum thin film coated on to a silicon wafer was imaged using a CAMECA ims-1280HR to acquire the SIMS image shown in Figure 1b. The same "SIMS" feature was also imaged using secondary electron microscopy (SEM) and is shown in Figure 1a. The two images were registered using the MATLAB Image Processing Toolbox by defining 8 control points common to both images. As can be seen, the SIMS image needed to be scaled differently in both the $\mathrm{x}$ - and $\mathrm{y}$ dimensions, requiring a projective transform to properly register the image pair. Pan-sharpening, a subset of image fusion that fuses a color image, in this case the SIMS image, with a higher resolution, 
panchromatic image, the SEM image, was used to produce the fused image seen in Figure 1c. This image benefits from an improved visual quality, combining the high spatial resolution information with the elemental/isotopic information encoded as color in the SIMS image.

In the previous example, confirming that the same area was analyzed using both techniques was straightforward, since the feature of interest was clear and distinct. However, for "real-world" systems, protocols need to be developed to ensure that the analysis area matches when using separate instruments. This may include a standardized coordinate system on the sample substrate or distinguishable features that are readily identifiable across imaging modalities. In the latter case, control point image registration could then be utilized to properly register the input image pair before performing image fusion.

The work presented here illustrates the implementation of image fusion with SIMS images acquired using a CAMECA ims-1280HR with higher resolution electron microscopy images. Ultimately, the goal is to continue to develop and refine the protocol outlined above using imaging standards so that the procedure can be applied to "real-world" systems where the elemental and isotopic features of interest lie below the current achievable lateral resolution of SIMS. [5]

\section{References:}

[1] X Huang, et al, IEEE Trans. Geosci. Remote Sens. 11 (2014), p. 714.

[2] J L Rubio-Guivernau, et al, Bioinformatics 28 (2012), p. 238.

[3] J G Tarolli, et al, J. Am. Soc. Mass Spec. 25 (2014), p. 2154.

[4] T Milillo, et al, Surf. Interface Anal. 47 (2015), p. 371.

[5] The research described in this paper was conducted under the Laboratory Directed Research and Development Program at Pacific Northwest National Laboratory, a multiprogram national laboratory operated by Battelle for the U.S. Department of Energy.
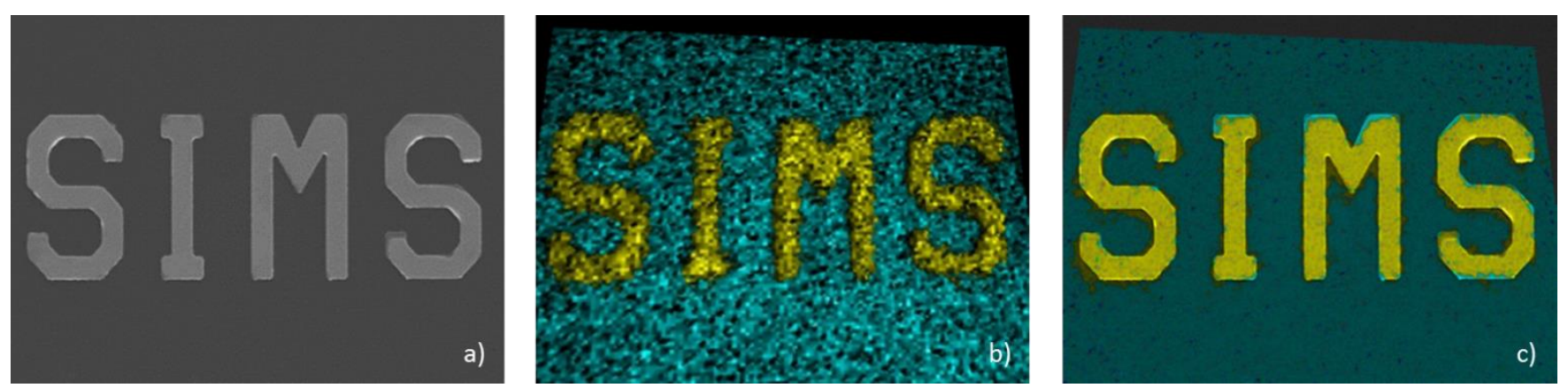

Figure 1. (a) SEM image of the word "SIMS" on an image standard; (b) SIMS image of the same feature where yellow represents the $\mathrm{Si}$ distribution and cyan represents the Ta distribution. The input images were registered using the MATLAB Image Processing Toolbox; (c) result of image fusion via pansharpening of (a) and (b). 